\title{
Sugerencias Psicopedagógicas para lograr una autoestima adecuada en los niños en situación de discapacidad.
}

\section{Psychological suggestions to achieve adequate self-esteem in children with disabilities}

DOI: $10.46932 / \mathrm{sfjdv} 2 \mathrm{n} 2-109$

Received in: March 1st, 2021

Accepted in: May 30th, 2021

\author{
Dr.C. Eddy Betancourt Escobar \\ Universidad de Ciencias de la Cultura Física y el Deporte. La Habana Cuba. \\ Correo: eddybe1967@gmail.com
}

\section{RESUMEN}

El nacimiento de un hijo con características especiales o distintas a la media, es un acontecimiento duro de aceptar por toda familia. El ideal de hijo esperado es de un niño hermoso físicamente y que sea la alegría de toda la familia como resultado de una de sus funciones vitales; se necesita estar preparados para poder irradiar alegría y recibirlos con amor para que sean educados en ese ambiente. El trabajo presentado refiere a la preparación que desde la escuela debe recibir la familia del niño con déficit en el desarrollo o necesidades educativas especiales, para que desde su situación de discapacidad puedan elevar su autoestima, siendo esta uno de los componentes psicológicos en la formación de la personalidad más dañados y estigmatizados en las personas que sufren algún déficit en su desarrollo. La propuesta presentada, es un pequeño aporte consistente en alternativas a tener en cuenta, para contribuir desde las ciencias pedagógicas en la preparación de los docentes y de las familias para incidir en el mejoramiento de la autoestima de los niños con estas características, propiciándoles un mejor estado de ánimo psicológico que les facilite afrontar la vida, sus retos en plena armonía y participación activa en la sociedad.

Palabras claves: Sugerencias, Autoestima, situación de discapacidad

\begin{abstract}
The birth of a child with special characteristics or mean differences is a hard event to accept in the family. The ideal of an expected child is of a beautiful child physically and that is the joy of the whole family as a result of one of his vital functions, it is necessary to be prepared to be able to radiate joy and receive them with love so that they are educated in that environment. The work presented refers to the preparation that the family should receive from the school with a deficit in development or special educational needs, so that from their situation of disability they can raise their self-esteem, being this one of the psychological components in the formation of the personality more damaged and stigmatized in people who suffer from a deficit in their development. The proposal presented is, a small contribution consists of alternatives to consider, to contribute from the pedagogical sciences in the preparation of teachers and families to influence the improvement of children's self-esteem with these characteristics, giving them a better psychological mood that makes it easy for them to face life, their challenges in full harmony and full participation in society.
\end{abstract}

key words: suggestions, Self-esteem, Disabilities 


\section{INTRODUCCIÓN}

La familia como célula fundamental de la sociedad, cumple importantes funciones, entre la que destaca: la función "Biosocial", con ella asegura la continuidad de la existencia humana. El nacimiento de un hijo, es el momento cumbre y esperado de toda familia, se reúnen, festejan, conversan, buscan parecidos, se reiteran las exclamaciones sobre lo hermoso y bello del recién nacido.

¿Pero qué ocurre en el interior de esa familia cuando el hijo tan esperado, presenta un déficit en el desarrollo o características especiales que no se corresponden con los sueños y las expectativas deseadas?

Para responder a esta interrogante, es necesario detenerse y evaluar las palabras de Vygotsky, cuando al referirse a los niños inválidos, plantea que generalmente se encuentran mucho más cerca del tipo normal de niño que los ciegos y los sordos y requieren mucho menos potencialidades en la Educación. Las dificultades relacionadas con la educación de los inválidos en gran parte son de carácter general (la imposibilidad de caminar en la escuela, de escribir, de trabajo etc.). La posibilidad de perder el equilibrio mental bajo la influencia de las dificultades y la posición socio psicológica especial creada por la posición exclusiva del niño invalido en el medio, son un peligro interno. Por eso la tarea de la educación consiste en prevenir el surgimiento de semejante sentimiento de inferioridad, desolación etc. Vygotsky (1989:165)

En el contexto educativo cubano, esta problemática ha sido abordada por muchos investigadores como, Marta Torres González (2002), Pedro Luis Castro Alegret (2003, 2005, 2008), Juana Bert Valdespino (2010) y Eddy Betancourt Escobar (2013), entre otros. Sus aportes son de mucha importancia, pues nos brindan información sobre el impacto socio psicológico que provoca en las familias el nacimiento de un niño con déficit en su desarrollo; además, ofrecen recomendaciones para la intervención psicopedagógica con estas familias y sus hijos. El niño recién-nacido con estas características hace que la familia pase por algunos momentos que son vitales en su dinámica y que cambia las relaciones personales, su actitud con los vecinos, con la comunidad y esto sin dudas no propicia una adecuada inclusión de estos niños en la sociedad.

Betancourt Escobar (2013), en su tesis doctoral explica los síntomas y las diferentes tendencias manifestadas por las familias de hijos con parálisis cerebral; describe las reacciones de los familiares desde el momento de la noticia hasta definitivamente la aceptación de la realidad. En muchas ocasiones, los padres carentes de orientación y acompañamiento psicopedagógico buscan refugio y explicación al problema de su hijo en fundamentos no científicos, relacionados con la mística y el credo popular; además, se observan sentimientos de culpa y responsabilidad, con un peso mayor en los roles de la madre, pues en muchos casos existe abandono y no reconocimiento por parte del padre.

El autor continúa explicando que la situación se agrava cuando la familia privilegia el cuidado y la alimentación del niño, dejando a la zaga elementos tan importantes como los paseos, las charlas, los juegos y las lecturas; lo que, sin dudas, influyen en el detrimento del desarrollo socio cultural del niño. A 
esta situación se le suma, la escasa orientación que los profesionales brindan a la familia para que puedan comprender que la discapacidad es un problema social y como tal debe ser atendido. Sobre lo anterior Vygotsky (1989:57) afirmaba "La deficiencia infantil constituye en la gran mayoría de las cosas, el producto de las condiciones sociales anormales..."

La Dra.C. Ana Luisa Sagarte Iznaga, en mesa redonda especial (1999) al referirse a la situación social del desarrollo del niño plantea, que es posible distinguir dos líneas fundamentales: una que tiene que ver con las condiciones externas en que la vida del niño transcurre y otra que tiene que ver con los procesos internos de desarrollo y destaca entre las condiciones externas la familia como institución fundamental y donde comienzan a formarse sus peculiaridades psicológicas; además de existir e influir otros como: el barrio y la escuela que son elementos socializadores. En los procesos internos de desarrollo pudiéramos señalar dos líneas fundamentales: el desarrollo intelectual y los procesos motivacionalesafectivos-morales íntimamente relacionados entre sí.

La Dra.C Elsa Gutiérrez Baró (1999). Plantea que los niños necesitan recibir amor y para el desarrollo de su personalidad el afecto es muy necesario, argumenta que es el nutriente espiritual más importante que existe para lograr una personalidad equilibrada y plena.

Arés Murzio, Patricia (1999) Plantea que hay un consenso de la mayoría de los psicólogos y psiquiatras sobre aquellos eventos y conflictos que puede vivir un niño en los primeros años de su vida y que pueden ser traumáticos, considerados eventos de alto riesgo. Estos son:

-La muerte de un ser querido, muy implicada a su cuidado, atención ya sus afectos

-Situación catastróficas o que les llaman desastres, que ya serían como hechos devastadores donde el niño tiene que movilizar cantidad de recursos adaptativos por encima de la violencia de los estímulos estresantes que se están presentando en un momento determinado.

-Aparece la privación de la familia como grupo

-El desarraigo o separar al niño de su ambiente o de su contexto, hasta situaciones de mudanzas, permutas, cambios de escuelas, peor aún, si es de forma tensionante o brusca.

Cuando no hay un entorno de confianza, cuando no hay un ambiente de seguridad, el niño no puede abrirse, no puede expresar sus inquietudes. Se conoce que la producción excesiva de estímulo con el niño, no es aconsejable, se cae en la sobresaturación de estímulos, el exceso de información. El exceso de estímulos evidentemente va a generar un embotamiento afectivo mayor; creando un estado de confusión que puede ser, para el desarrollo del niño, una circunstancia de vulnerabilidad psicológica muy peligrosa. Los padres deben ser ayudados por el personal especializado para evitar la aparición en los niños con algún déficit en su desarrollo, de los llamados "defectos secundarios": físicos, sociales y psicológicos, siendo los dos últimos por lo regular, los que ocasionan más daños que los biológicos y los 
físicos; lo anterior fue bien explicado por Vygotsky (1989:57) en la estructura del defecto y que acuñando este tema planteó “'La deficiencia infantil constituye en la gran mayoría de los casos el producto de las condiciones sociales anormales....

Está demostrado por estudios socio- psico-pedagógicos, que la llegada de un hijo con déficit en el desarrollo provoca trastornos y desequilibrio en todas las áreas y afecta la dinámica familiar. Aparecen sentimientos de culpa, no reconocimiento y hasta el rechazo por el recién nacido; se sugiere la atención integral de todos los factores para potenciar y empoderar a estas familias.

Debido a lo que acontece en el interior de estas familias y sobre todo a la educación que debemos ofrecer a los niños con déficit en el desarrollo, es que surge la necesidad de este trabajo, dirigido a potenciar la autoestima de las familias afectadas y de los niños en esta situación, con la seguridad que todos pueden y tienen potencialidades de desarrollo. Para esto es necesario su formación integral, que los incluiría sin prejuicios y de forma útil en la sociedad.

En el desarrollo de la personalidad juega un papel esencial el propio concepto que tenga de sí mismo el sujeto, cómo se valora, se respeta y se ama así mismo. Solo así podrás valorar, respetar y amar a los demás, siendo esto muestra de equilibrio y ajuste emocional.

La necesidad de potenciar la autovaloración y la confianza en sí mismo, ha sido objeto de preocupación desde siglos atrás y es así como psicólogos, sociólogos y pedagogos se han pronunciado en tal sentido; para Vygotsky y sus discípulos, el problema fundamental del niño con déficit en el desarrollo físico motriz, es lo relacionado a su sensación de soledad y autoestima. Sobre esta situación expresó “No debemos poner al niño en posiciones difíciles que lo hagan sentir inferior a los demás“ y continua el autor: para A. Adler y su escuela, la lucha contra la soledad y el sentimiento de minusvalía es el punto de partida y fundamental de toda la educación“. Vygotsky (1989:160)

Bozhovich, en su investigación sobre las motivaciones de la conducta de los niños y adolescentes expresó: "La actitud que se va formando en el niño, hacia sí mismo, su autovaloración y su nivel de aspiraciones, depende de cómo hace frente a todas esas tareas y de la actitud que adoptan hacia él, los individuos que lo rodean y de cómo valoran estos sus actos. Las exigencias sociales son asimiladas por el niño y se convierten en sus propias exigencias, hacia sí mismo“. (1961:138)

Según criterio de especialistas en el tema y de muchos psicólogos, la autoestima baja, está considerada como una de las causas que más incide en los intentos suicidas en las personas; la organización Mundial de la Salud (OMS) estima que cada día al menos 1000 personas se suicidan y 10 000 lo intentan. El suicidio se encuentra entre las 10 primeras causas de muerte en la mayoría de los países. 
El destacado pedagogo e investigador cubano, Gustavo Torruella (1999), preocupado por esta situación en su artículo: “La autoestima, clave del éxito personal" expresó “... Una meta principal de la educación debería ser el formar en los niños y jóvenes una autoestima positiva, una valoración de las propias potencialidades, porque uno irá tan lejos en el viaje de la vida como crea que es capaz de hacerlo."

Para el citado autor, el aspecto más importante de la personalidad de cada ser, es el concepto y la valoración que tenemos de nosotros mismos; insiste en que la autoestima, es la base de todo crecimiento y desarrollo personal, es el coraje de ser uno mismo y de confiar en los propios recursos. Argumenta, que existen dos tipos de personas: aquellas que tienen un concepto positivo de sí mismas, es decir que poseen una alta estimación y una buena imagen de sí, que tienen confianza y fe en sus recursos, capacidades y se sienten competentes para alcanzar las metas que se proponen. Estas personas tienden a triunfar y lograr altas metas.

La otra clase de persona es la que tiene un concepto deficiente de sí misma, carece de confianza en sus recursos y capacidades y se siente incompetente para emprender nuevas tareas. En general obtienen solo pobres logros y pequeñas metas.

Torres González (2003), plantea que la autoestima es una construcción psicológica que depende en su formación inicial de la valoración externa, la aprobación o desaprobación, aceptación o rechazo, protección o abandono, compañía o soledad, afectos o desafectos de los otros. En primera instancia por los padres y después, de acuerdo al ciclo evolutivo de la vida, por los otros adultos, los maestros, el grupo escolar, los amigos, el colectivo laboral, la pareja, los dirigentes, los hijos, la sociedad y define la autoestima como: "la fuerza motriz del desarrollo y del comportamiento humano que implica un nivel afectivo, relacionado con la aceptación que tienes de ti mismo, cuanto te quieres, como eres y el grado de satisfacción personal de ser asî” (2003:53)

Cada individuo va aprendiendo a quererse o no, a aceptarse, sentirse conforme consigo mismo, saber que es él y no otro, que tiene identidad propia. A conocer y reconocer sus posibilidades, cualidades a sentir que es y está. Ese aprendizaje es afectivo, cuando en su interacción con su contexto familiar ha sentido que lo quieren, lo respetan, que es importante para sus padres, abuelos, hermanos, pero que siempre lo es. Las buenas relaciones y convivencia familiar tienen una gran significación para la formación de una elevada autoestima; en dependencia de la alternativa que asuma estaremos en presencia de: Autoestima elevada, Autoestima media, Autoestima baja y Autoestima muy baja.

La autoestima constituye una fuerza motriz del desarrollo y del comportamiento humano, la autoaceptación de cada individuo promueve el yo puedo, yo lo lograré, venceré, me realizaré, puedo aportar, ayudar a servir a los otros, puedo cometer errores; la ausencia de la figura de los padres, la falta o pobre contacto afectivo, el rechazo abierto o encubierto, la continua desvalorización y descalificación. 
El contacto con actitudes pesimistas, negativas, llenas de resentimiento, rabias y rencores, generalmente producen una disminución de la autoestima, lo que repercute negativamente en su proyecto de realización personal.

En la definición de Autoestima emitida por algunos autores, se observan coincidencias y puntos de encuentros en declarar que responde al nivel de aceptación que tiene la persona de sí misma, de confiar en sus posibilidades para enfrentar las necesidades que se presentan. Otros, plantean que la autoestima es una construcción personal que tiene raíces muy tempranas. Es expresión de autoconfianza y seguridad en nosotros mismos. Es un concepto dinámico que varía y se enriquece en los diferentes momentos, espacios y contextos en que se producen nuestras interrelaciones.

Parreño Montero, W (2007) ofrece una definición de autoestima en la que agrega algunos elementos que la distingue de los anteriores autores, explica que no solo por la valoración que tenemos de sí mismo; es aquella que se va adquiriendo y por la que nos dan los demás, los que nos rodean, ubicando a la familia en primer lugar. La autoestima adecuada se convierte en gran medida en responsable de muchos éxitos y fracasos.

La autora ofrece algunas recomendaciones a considerar para elevar la autoestima que sugerimos sistematizar con docentes y familias para el caso que nos ocupa: no establecer comparaciones, exaltar y estimular los logros de nuestros niños, sus aspectos positivos, capacidades, habilidades etc., estar prestos a orientarlas y brindarles nuestra ayuda cuando lo soliciten y necesiten, demostrar que cada persona puede desempeñarse con éxito en una esfera determinada, motivarlas y orientarlas.

Según su opinión, para lograr una autoestima adecuada, debe corresponderse con nuestras posibilidades reales. No debemos pensar que lo sabemos todo y que todo lo hacemos perfectamente, pero tampoco que lo hacemos todo mal y que somos incapaces de obtener éxito en alguna actividad.

Humberto González Rosario (2007) Propone un manual para la prevención del VIH-SIDA “Canto a la vida y a la esperanza“ En el mismo hace énfasis en la necesidad que tiene el individuo de aprender a conocerse para darse cuenta de todas las posibilidades que están dentro de ti; el referido autor define la Autoestima como recurso psicológico que nos ayuda a enfrentar situaciones difíciles, asumir riesgos, ser creativos, productivos, emprendedores, tomar decisiones, tener relaciones familiares, escolares y sociales efectivas. Reconoce que una persona con autoestima tiene mayores posibilidades de decir lo que le conviene y de rectificar cuando ha tomado una decisión inadecuada; el autor incluye un elemento nuevo en relación con la autoestima, que es de consideración atenderlo y responde a la Autoimagen definida por el como la imagen que tienes de ti mismo.

El destacado profesor e investigador de la psiquiatría infantil cubano Cristóbal Martínez Gómez, en mesa redonda especial (1999) Plantea que la principal fuente de satisfacción de las necesidades de un 
niño es la familia y en la medida en que esta satisfaga sus necesidades, el niño va a tener un desarrollo psicológico adecuado. Este investigador plantea que la mayor necesidad en las edades tempranas de un niño, es la necesidad de afecto; el niño necesita sentirse querido, que la gente lo quiera, agrega que muchos científicos al referirse al tema lo abordan como: "Hambre de afecto primario", dado en aquellos niños que reciben poco cariño, pocos afectos, lo que genera trastornos en su personalidad; este investigador nos explica lo que el niño necesita. Seguridad, aceptación, cuidados, ambiente estable, socialización, autonomía del juego.

Además, nos alerta del perjuicio que provoca otro elemento llamado "Sobreprotección”, y lo define como: el exceso de afectos, una exageración de la actitud afectuosa normal (conducta desmedida de las madres en devoción por sus hijos, que no permiten que nada se interponga en sus deberes maternales y así reducen las actividades en otros aspectos de la vida y las divide en dos grandes grupos: las puras y las culpables compensadoras: las puras, son mujeres que muestran excesivo cuidado hacia sus hijos por verdadera devoción y las culpables compensadoras: son mujeres que protegen extraordinariamente a sus hijos para ocultar o compensar actitudes hostiles o de rechazo de las que generalmente no se percatan.

Otro aspecto a considerar es al referido a la permisividad, el niño recibe todo lo que quiere siempre que sea económicamente posible y se le deja hacer todo lo que desea, los hijos de madres excesivamente afectuosas o sobreprotectoras son muchas veces permisivos. Exceso de ansiedad: se ve cuando ha existido una enfermedad grave o una muerte entre los hijos. Es muy frecuente en familias de un solo hijo, se asocia

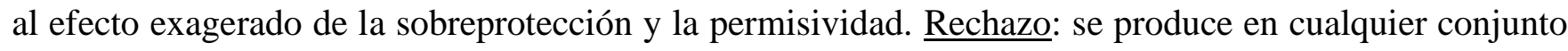
de circunstancia en que el hijo no sea deseado, sin embargo, los hijos no deseados pueden llegar a ser amados y los hijos deseados a veces son rechazados. Por lo regular las madres que rechazan y abandonan a sus hijos son inmaduras, inestables o neuróticas debido a que fueron rechazadas cuando niñas.

Características de los padres en esta situación

-Discordia: falta de afecto, la aversión a la gestación al matrimonio forzado, oposición al matrimonio por familias en el pasado, interrupciones de estudios y proyectos por ese matrimonio forzado. Por lo general cuando algún niño presenta algún déficit en el desarrollo es más frecuente el rechazo al igual que por el tipo de sexo que no es el deseado y puede ser un rechazo abierto o encubierto por esfuerzos conscientes o inconscientes.

La conducta de los padres hacia sus hijos se caracteriza por: Severos castigos, regaños, desprecios, indiferencias, irritación, amenazas tratos incoherentes, suspicacia, resistencia a gestos y desfavorables comparaciones entre los demás hermanos. El defecto de personalidad que más sobresale en el niño rechazado es la falta de un sentimiento de pertenencia. Cuando el rechazo de los padres es abierto generalmente el hijo reacciona con agresividad y deseos de tomar represalias, mezclado con infelicidad. 
Exceso de autoridad: Identificación, Vuelven a vivir su vida con sus hijos y le imponen las cosas que ellos no pudieron ser y quieren que sus hijos lo logren (deportistas, bailarines, músicos etc.)

-Perfeccionismo: lograr la perfección en todo lo que se haga, consejo instrucciones, sugerencias. Se le da poco crédito al niño y a su aprovechamiento. La presión sobre el hijo para que logre buenos resultados es normal en estos casos.

Exceso de crítica: Los padres tratan a sus hijos como un ser inferior, lo ridiculizan, humillan. Se les lastima su orgullo y pierde la estimación en sí mismo. Se siente inferior ante sus compañeros, infeliz, tímido, e insatisfecho.

Tensión emocional: hiperactividad, hábitos nerviosos, retraimiento, volverse indebidamente dócil y tímidos y reaccionar con conductas agresivas y desafiantes.

Inconsistencia: Lo disciplina para que tengan éxitos, para que sea aceptado, supone que por parte de los padres exista una clara conciencia de las necesidades del niño para madurar y el respeto que ello implica. El niño debe tener consideración hacia otro y a respetar la propiedad ajena.

Torres Gonzales 2003:87, nos ofrece elementos que desde la psicología presentan los padres que lo hacen ser más racional para enfrentar la discapacidad de sus hijos: nivel de funcionabilidad, grado de cohesión de la pareja, mayor o menor tolerancia a la frustración, mayor o menor nivel de dependencia de la opinión y aprobación social, sentido de responsabilidad, autoestima y seguridad.

Según la autora, la regla de oro en la orientación familiar consiste en que el maestro tiene que continuamente autoevaluar sus actividades hacia la familia y los alumnos, no puede dejarse influir por la subjetividad, la empatía, el carisma de una familia y quiera que sus logros sean lo de la otra; debe aceptar la diversidad familiar de todos sus iguales. Continúa planteando la investigadora, que uno de los principales factores protectores de la familia es su autoestima, cuando está disminuida, aumentan las posibilidades de riesgo o se agravan; al no aceptarse como tales y al no quererse como familia, es muy difícil producir el respeto, la consideración, la estimación del entorno.

En estos casos aparece un efecto de rebote, porque al sentirse rechazados, marginados, valorados como conflictivos, refuerza la autodesvalorización, la desmoralización y con ello la disminución de la autoestima como grupo y como individuos. Acentúa la pobreza de identidad y asumen conductas que pueden comprometer su ajuste social y personal.

Las consideraciones de la autora son necesarias tenerlas en cuenta, ya que es en familia donde se reciben las primeras enseñanzas y es la primera escuela del niño; Además, nos ofrece algunas sugerencias para lograr una autoestima elevada en las familias y en sus integrantes independientemente de las características individuales que debemos tener en cuenta y respetar.

-Satisfacción de las necesidades individuales, del grupo familiar y de la pareja 
-Desarrollar una filosofía del valor, del estímulo, de la aprobación, la crítica constructiva, el respeto, el apoyo y la confianza.

-Evitar las conductas y las verbalizaciones que descalifiquen y desvalorizan. Concentrarse en lo positivo, en las potencialidades, no en lo negativo, en el defecto.

-Promover una actitud optimista y decidida ante la cotidianidad y ante los acontecimientos vitales.

-Cultivar el principio de la aceptación de unos a otros, sin negociación de los afectos.

-Plantearse metas y expectativas individuales y colectivas de acuerdo a las posibilidades de realización.

-Luchar por la estabilidad de la estructura familiar

-Mantener el contacto afectivo y efectivo aun en casos de disolución matrimonial.

-Evitar la comunicación irónica, los dobles mensajes, permeados por rabia, culpa y rencores.

-Crear un intercambio agradable para el intercambio familiar.

-Prevenir actitudes de preferencias y privilegios de unos miembros con respecto a otros.

-Promover la espontaneidad y creatividad individual y colectiva

-Estimular y desarrollar el sentido del humor y compartirlo en familia.

-Criticar y evaluar las acciones negativas.

El autor es del criterio que estas sugerencias se deben tener en cuenta por maestros y especialistas ya que tienen un gran valor Psicopedagógico para el trabajo con las familias y en particular para elevar la autoestima en los niños con algún déficit en el desarrollo y en muchos casos, la de los propios padres.

\section{CONCLUSIONES}

La revisión bibliográfica y el intercambio con algunos especialistas sobre el tema, nos permitió arribar a la siguiente consideración: la baja autoestima provocada por la no aprobación, el ridículo constante, la exclusión al no hacerles partícipes en las conversaciones y toma de decisiones; suele expresarse mediante mecanismos compensatorios que muestren lo contrario y se convierten en escudos protectores o refugios, ejemplo: la enajenación, irritación constante y lo que es peor en otros casos muestra de superioridad, machismo; asumiendo conductas llamativas para lograr convertirse en el centro de atención.

La propuesta en esta investigación consiste en sugerencias psicopedagógicas para orientar a las familias y docentes en el trabajo por lograr una autoestima adecuada en los niños y personas con déficit en su desarrollo o situación de discapacidad, la misma puede ser tenida en cuenta para su aplicación en los hogares de las familias en esta situación o en el contexto escolar. El logro de una autoestima adecuada en los niños con déficit en el desarrollo o en situación de discapacidad, depende de la Educación oportuna que puedan ofrecer los adultos que lo rodean en la primera infancia, donde ocupa un lugar insustituible la 
familia y la escuela; al respecto, Vygotsky (1989:114) sentenció: “El desarrollo insuficiente de las funciones superiores está relacionado con el desarrollo cultural insuficiente del niño retrasado mental, con su desaparición del medio cultural circundante y el abandono de la alimentación y del medio.... 


\section{BIBLIOGRAFÍA}

Aréz Mursio, Patricia. (2002). Mi familia es así, Ed. Pueblo y Educación, Ciudad de La Habana, Cuba. Betancourt Escobar, Eddy (2013) La orientación familiar para la educación de los niños con necesidades educativas especiales por parálisis cerebral. Tesis presentada en opción al título de Doctor en Ciencias Pedagógicas. Universidad de Ciencias Pedagógicas de Holguín, Cuba.

Castro Alegret, Pedro Luis (2004). El maestro y la familia del niño con dificultades. Reino Unido. Editorial Save the Children.

Pueblo y Educación. Cuba.

(2008). El maestro y la familia del niño con discapacidad. La Habana. Editorial

Constitución de la República de Cuba (2019)

Fernández, Gudelia. (2008). La estimulación Temprana. Editorial Pueblo y Educación. La Habana. Cuba.

García Morey, Aurora y Elsa Gutiérrez Baró (1999) ¿En qué tiempo puede cambiarse la mente de un niño? Mesa Redonda. Edición especial: casa editora abril. La Habana, Cuba.

Martí Pérez, José (2000). Ideario Pedagógico. Ed. Pueblo y Educación, La Habana.

Martínez Gómez, Cristóbal (2001). Salud Familiar, Editorial. Científico Técnica. La Habana, Cuba.

Parreño Montero Wally (2007). Autoestima. Algo para cultivar. Revista Educación. No. 121 mayo agosto, 2007/segunda época. La Habana. Cuba.

Ravelo Aloima, (2011) ¿Cómo educar a los hijos sin equivocarnos tanto? Ed. Pueblo y Educación, La Habana.

Torres González, Martha. (2003). Familia, Unidad y Diversidad. Ed. Pueblo y Educación, La Habana, Cuba.

Torruella, Gustavo. (1999). La autoestima: clave del éxito personal. Conferencia ofrecida en el diplomado ". Aprendizaje básico del desarrollo humano. IPLAC,

MINED, La Habana, Cuba.

Turner, Lidia y Balbina Pita. (2002). Pedagogía de la Ternura. Ed. Pueblo y Educación, La Habana, Cuba. Vigotsky, L.S. (1989). Obras completas. Ed. Pueblo y Educación. La Habana, Cuba. 